\title{
Optimization of Wall Compon ents for Encapsulation of Nigella Sativa Seed Oil by Freeze-Drying
}

\author{
Sina Makouie ${ }^{1}$, Mohammad Alizadeh ${ }^{*}$, Omid maleki ${ }^{1}$, Asghar Khosrowshahi ${ }^{1}$ \\ ${ }^{I}$ Food Science and Technology Department, Agriculture Faculty, Urmia University, Urmia, Iran \\ (*) Corresponding Author \\ E-mail:malizadeh@outlook.com \\ Tel: +989143474341
}

\begin{abstract}
The aim of the present study was to determine the proper concentrations of whey protein isolate (WPI), Arabic gum (AG), and Zedo gum (ZG) for the encapsulation of Nigella sativa seed oil (NSO). WPI, AG, and ZG were used as wall materials for the encapsulation of NSO by mixture design and then the optimum sample with minimum Z-average, PDI, and a* and maximum zeta potential, conductivity, encapsulation efficiency, and $b^{*}$ was selected for examining FT-IR and SEM. The findings demonstrated that lower particle size and particle dispersion index were obtained at higher amounts of AG and WPI with the least a*. Desirability function results indicate that the mixture of $39.05 \%$ WPI, $16.758 \% \mathrm{AG}$, and $4.192 \% \mathrm{ZG}$ led to the production of the optimum sample. Microencapsulation of Nigella sativa seed oil using the optimum wall composition was recognized to be useful to preserve bioactive compounds and provide flavor stability, enabling the use of microcapsules in food formulations.
\end{abstract}

Keywords-Nigella sativa seed oil, Whey protein isolate, Arabic gum, Zedo gum, FT-IR, SEM

\section{INTRODUCTION}

As an annual herb belonging to the Ranuculacea family, Nigella sativa L. is consumed for edible and medical purposes. It has been reported that its oil possesses antioxidant, anti-inflammatory, antitumor, and antimicrobial activities. It has also used to treat illnesses like asthma, bronchitis and emphysema [1,2]. Phenolic compounds existing in Nigella sativa, including thymoquinone, carvacrol, t-anethole and 4-terpineol have considerable free radical scavenging properties [3]. Since such these natural antioxidants are very sensitive compounds, they should be preserved from environmental conditions such as oxygen, water, and light in order to demonstrate its pharmaceutical features. In the field of food processing, Encapsulation is a practical technique to retain bioactive compounds during food storage. In addition, encapsulation leads to controlled release during consumption, prevention of undesirable interactions of bioactive compounds with the food matrix, and extension of shelf life of the product. Freeze-drying technique was used to encapsulate N. sativa seed oil since it is very suitable for conservation heat sensitive compounds which almost remain undamaged the initial functional properties of those components [4]. Possession of high water solubility, low cost and viscosity, film-forming and emulsifying properties are essential factors art choosing suitable wall material for successful encapsulation [5]. The encapsulating agents used in this study were Zedo Gum $(\mathrm{ZG})$, Whey Protein Isolate (WPI) and Arabic Gum (AG).
Arabic gum (AG) is the most common wall material for encapsulation of oils and bioactive volatile compounds [6]. Whey protein isolate (WPI), as a nutritional ingredient, is greatly used in the food industry because of its emulsifying, gelling and foaming properties [7]. WPI has excellent surface activity due to its mixed composition of $\beta$ lactoglobulin, $\alpha$-lactalbumin, bovine serum albumin and other proteins, such as casein [8]. Zedo gum (ZG) which is also known as Angum, is an anionic and acidic hydrocolloid which exudes from Amygdalus scoparia Spach tree [9]. It is consumed as a functional ingredient for nutritional and pharmaceutical purposes such appetite stimulant and crushing bladder stones.

The objective of this study was to evaluate the potential of GA, WPI, and ZG for encapsulation of $N$. sativa seed oil. This study investigated the impacts of the different concentrations of wall materials in various core/wall ratios $(1 / 1.5-1 / 4)$ on encapsulation efficiency and other physical properties of $\mathrm{AG} / \mathrm{WPI} / \mathrm{ZG}$ microcapsules loaded with $\mathrm{N}$. sativa seed oil. The optimal composition of wall materials were determined to produce N. sativa seed oil microcapsules. Finally, the structural and morphological properties of microcapsules produced in optimized core/wall ratio and concentration of $\mathrm{AG}$, WPI, and $\mathrm{ZG}$ were characterized by Fourier transform infrared spectroscopy (FTIR) and scanning electron microscopy (SEM) analyses. 


\section{MATERIALS AND METHODS}

\section{A. Materials}

Cold pressed Nigella sativa seed oil was purchased from a local market (Urmia, west Azerbaijan, Iran). Gum Arabic and Whey Protein Isolate were obtained from Merck (Darmstadt, Germany). Zedo Gum was purchased from Freer flavor and color knowledge-based company (Isfahan, Iran). All other reagents were provided from Sigma-Aldrich (Gillingham, Dorset, UK). Distilled water was used in all experiments.

\subsection{Encapsulation process}

The wall materials were added to distilled water at $25 \mathrm{C}$, and the mixture was stirred magnetically until completely dissolved. The solution was left overnight at
$4 \mathrm{C}$ to allow full hydration. The total solid concentration

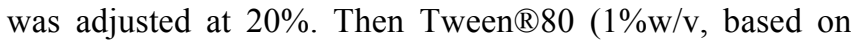
water) was dissolved in the mixed wall material solution. $N$. sativa seeds oil was then added to the wall material solution based on the experimental design (Table 1). After the whole mixture stirred magnetically for 5 minutes, the formed coarse emulsion was homogenized using an Ultra-Turrax homogenizer (IKA-T18 Digital) operating at $3000 \mathrm{rpm}$ for 10 minutes to produce pre-emulsion. Lastly, the preemulsion was further homogenized for 10 minutes at full sonicator power (200.0 Watt, $24 \mathrm{kHz})$ using ultrasound homogenizer (UP200; Hielscher, Teltow, Germany) equipped with a titanium probe of $14 \mathrm{~mm}$ in diameter. The prepared emulsion used as the feed liquid for the freezedrying process.

TABLE 1.

MIXTURE DESIGN USED FOR EXPLORATION OF OPTIMUM WALL COMPOSITION FOR ENCAPSULATION OF NIGELLA SATIVA SEED OIL.

\begin{tabular}{ccccc}
\hline Run & $\begin{array}{c}\text { Component } 1 \\
\text { A: WPI }(\%)\end{array}$ & $\begin{array}{c}\text { Component } 2 \\
\text { B: Zedo gum }(\%)\end{array}$ & $\begin{array}{c}\text { Component 3 } \\
\text { C: Arabic gum }(\%)\end{array}$ & $\begin{array}{c}\text { Component } 4 \\
\text { D: Oil (\%) }\end{array}$ \\
\hline 1 & 60 & 20 & 0 & 20 \\
2 & 26.7 & 0 & 53.3 & 20 \\
3 & 0 & 10 & 60 & 30 \\
4 & 0 & 10 & 60 & 30 \\
5 & 60 & 0 & 0 & 40 \\
6 & 20 & 6.7 & 33.3 & 20 \\
7 & 0 & 0 & 60 & 20 \\
8 & 0 & 0 & 80 & 20 \\
9 & 80 & 20 & 0 & 40 \\
10 & 0 & 20 & 40 & 30 \\
11 & 26.7 & 0 & 13.3 & 30 \\
12 & 35 & 0 & 35 & 20 \\
13 & 35 & 10 & 35 & 20 \\
14 & 35 & 10 & 35 & 40 \\
15 & 35 & 0 & 35 & 30 \\
16 & 0 & 10 & 60 & 20 \\
17 & 50 & 20 & 0 & 25 \\
18 & 50 & 0 & 0 & \\
19 & 53.3 & 15 & 26.7 & 45 \\
20 & 15 & & & \\
\hline
\end{tabular}

\subsection{Color analysis}

The color of the prepared emulsions was measured via a reflectance Minolta colorimeter (CR-400; Minolta, Tokyo, Japan). The material was spread over a plate, and four measurements were taken in random locations. The color parameters were expressed as $\mathrm{L}^{*}$ (lightness), $\mathrm{a}^{*}$ parameter (green-red), and $\mathrm{b}^{*}$ parameter (blue-yellow). Chroma (C) was estimated by $a^{*}$ and $b^{*}$ values. The following equation was applied to convert $L^{*} a^{*} b *$ coordinates to $C$ :

$\mathrm{C}=\sqrt{a^{* 2}+b^{* 2}}$

\subsection{Emulsion droplet size}

The size distribution and average diameter of the emulsion droplets were measured by the light-scattering technique using laser diffraction (Master sizer 2000; Malvern Instruments Ltd, Malvern, UK). The De Broukere mean diameter $\left(\mathrm{D}_{43}\right)$ and polydispersity index (PDI) of the microcapsules were determined by the method used by Karimi sani et al. in 2019 [10]. The samples were analyzed in triplicate using the wet method through dispersion in water and a refractive index of 1.52. All measurements were carried out at ambient temperature [11]. 


\subsection{Zeta potential}

One of the reliable indicators to predict the stability of emulsion systems is Zeta potential or surface charge measurement. In order to determine this quantity, the Zeta sizer instrument (ZS model, Malvern, UK) was used that operates based on the electrophoretic displacement of surface charged particles [12].

\subsection{Electrical conductivity}

Electrical conductivity of the samples was measured using digital electrical conductivity (Mod 8306, Italy) at ambient temperature.

\subsection{FT-IR analysis}

The structure analysis of the GA, WPI, and ZG polymers was examined by FT-IR. First, the $\mathrm{KBr}$ spectrum was obtained as a control. Preparation of samples for FT-IR spectroscopic analysis was carried out by mixing $1 \mathrm{mg}$ of completely dried sample with $150 \mathrm{mg}$ of dry $\mathrm{KBr}$ powder. A thin pellet of each sample was generated by compression the mixture with about $60 \mathrm{kPa}$ for 10 minutes in a compression instrument. To detect the structural composition of films incorporated with GA/WPI/ZG/NSO reinforcements, FT-IR spectroscopy (Spectrum two; Perkin Elmer, Waltham, MA, USA) in the wavenumber range of $4000-400 \mathrm{~cm}^{-1}$ with a resolution of $0.5 \mathrm{~cm}^{-1}$ was used [13].

\subsection{Surface morphology of microcapsules}

Scanning electron microscopy (SEM) was used to evaluate the surface morphology and the microstructural properties of the freeze-dried encapsulated powder produced in optimized conditions. Samples were coated with a thin conductive gold layer and then viewed using a scanning electron microscope (KYKY-EM3200) at an accelerating voltage of up to $20 \mathrm{kV}$.

\subsection{Encapsulation efficiency}

The encapsulation efficiency (EE) is characterized as the ratio of the oil inside the encapsulating wall material to the total oil present in the microparticles (coated + free). The amount of encapsulated NSO in the microcapsules was evaluated with the method used by Silva et al., 2014. In order to measure that, the surface oil of the powder was determined after producing by freeze drying. $4 \mathrm{~g}$ of powder was placed in a filter paper and washed six times with $10 \mathrm{ml}$ n-hexane. Then, the solution containing the extracted oil was left to evaporate at room temperature. The surface oil was calculated based on the difference between the initial clean flask and that containing the extracted oil residue, and the microencapsulation efficiency was calculated from the following equation [14];

$\mathrm{EE}=\frac{\left(W_{T O}-W_{S O}\right)}{W_{T O}} \times 100 \%$

Where:

EE: Encapsulation efficiency of N. sativa seed oil

TO: Weight of the total oil calculated in a sample of powder (based on the initial formulated emulsion)

SO: Weight of extractable surface oil experimentally found in the same weight of powder

\subsection{Statistical analysis}

A mixture design with 4 components was used for preparation of microcapsules. Different physicochemical responses were measured and then fitted to Scheffe models using least square method. Adj-R2 was used as a criteria of model selection. Analysis of variance and Fischer distribution were used for inference about statistical significance of models. Level of type I error was 0.05 . Design-Expert V 10, was used for data analysis and plotting of contour plots.

TABLE 2.

PREDICTIVE MODELS OBTAINED FROM MIXTURE DESIGN (MODEL TERMS IS ACCORDING TO TABLE 1).

\begin{tabular}{|c|c|c|}
\hline Variable & $\mathbf{R}_{2}$ & $\mathbf{R}_{\mathrm{adi}}{ }^{2}$ \\
\hline Z-average $($ d.nm $)=(457.86 * A)+(814.97 * B)+(1156.04 * C)+(484.60 * \mathrm{D})+(9673.79 * A B)$ & 0.61 & 0.49 \\
\hline $\begin{array}{l}\text { Zeta potential }(\mathrm{mV})=-(22.36 * \mathrm{~A})+(95.27 * \mathrm{~B})+(2.56 * \mathrm{C})-(465.5 * \mathrm{D})-(120.2 * \mathrm{AB})- \\
(47.05 * \mathrm{AC})+(551.43 * \mathrm{AD})-(210.5 * \mathrm{BC})+(503.04 * \mathrm{BD})+(489.41 * \mathrm{CD})\end{array}$ & 0.93 & 0.87 \\
\hline Encapsulation efficiency $(\%)=(76.59 * A)+(64.51 * B)+(94.89 * C)+(73.21 * D)+(98.24 * B C)$ & 0.94 & 0.91 \\
\hline $\mathrm{PDI}=(0.7769 * \mathrm{~A})+(2.11 * \mathrm{~B})+(0.5214 * \mathrm{C})+(0.19 * \mathrm{D})$ & 0.60 & 0.51 \\
\hline $\begin{array}{l}\text { Conductivity }=(0.34632 * \mathrm{~A})-(0.20018 * \mathrm{~B})+(0.30008 * \mathrm{C})-(0.33352 * \mathrm{D})+(2.84866 * \mathrm{AB})+ \\
(0.03047 * \mathrm{AC})+(0.47028 * \mathrm{BC})-(8.95829 * \mathrm{ABC})\end{array}$ & 0.91 & 0.83 \\
\hline $\mathrm{a}^{*}=-(8.07448 * \mathrm{~A})-(3.53583 * \mathrm{~B})-(6.31957 * \mathrm{C})-(7.61198 * \mathrm{D})$ & 0.92 & 0.90 \\
\hline $\mathrm{b}^{*}=\left(15.45^{*} \mathrm{~A}\right)+(24.6 * \mathrm{~B})+(12.53 * \mathrm{C})+(12.97 * \mathrm{D})$ & 0.48 & 0.36 \\
\hline Chroma $=(17.4327 * \mathrm{~A})+(24.8528 * \mathrm{~B})+(14.0334 * \mathrm{C})+(15.0387 * \mathrm{D})$ & 0.94 & 0.94 \\
\hline
\end{tabular}




\section{RESULTS AND DISCUSSION}

\section{A. Characterization of particle size distribution}

The results showed that the change in the concentration of the wall materials had a significant effect on the particle size of the produced microcapsules $(\mathrm{P}<0.05)$. Moreover, the predictive models for particle size as a function of the investigated factors are illustrated in Table 2. Figure 1 shows that by increasing the Whey Protein Isolate, the average particle size of the microcapsules was reduced. This finding could be attributed to the effective emulsifying properties of WPI and several hydrophobic and hydrophilic parts of WPI [15]. Thus, WPI has a higher capability to adsorb at the oil-water interface, and can reduce interfacial tension.

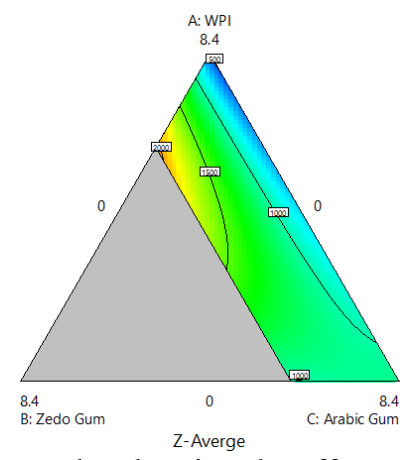

Fig. 1. Contour plot showing the effects of wall materials on the microparticles size distribution of encapsulated oil at $30 \%$ oil level.

Furthermore, increasing the amount of Arabic Gum led to microcapsules with a smaller particle size. Arabic Gum owns emulsifier property due to the presence of some peptide branches in its structure, these peptide branches might have attached to the droplets of oil contained in the emulsion and produced smaller particles [16]. In addition, by increasing Zedo Gum, the average particle size of the microcapsules was enhanced. This effect can be attributed to the greater Zedo Gum concentration that could potentially provide a higher viscosity and larger particles might be formed due to the agglomeration $[17,18]$.

\section{B. Surface charge stability}

Surface charge stability ( $\zeta$ potential) determines the surface charge at the interface of the droplets, it can be anionic, cationic, or non-ionic based on the kind of the charge of the surfactants that absorbed on the droplets. Zeta potential of the emulsion system is considered to be stable at the range of $30-60 \mathrm{mV}$ [19]. In this study, the absolute values for the zeta potential of emulsions were measured to be around $30 \mathrm{mV}$. The results showed that the change in the concentration of the wall materials affected on the zeta potential of the microcapsules significantly $(\mathrm{P}<0.05)$.

The presence of a negative charge on the microcapsules was intensified by raising the amount of WPI, while in case of $\mathrm{AG}$ it was reversed. By increasing the Whey Protein Isolate, the zeta potential of the microcapsules in all three levels of the oil was increased. This might be due to the net negative charge of the WPI at $\mathrm{pH}$ values greater than their isoelectric $\mathrm{pH}$. Plus, it can be attributed to the proper protein coating, in which case the negative repulsive force of the particle surface increases, and eventually the zeta potential gets more negative.

Moreover, increasing the amount of Arabic Gum in 20 and 30 percent of oil (Figure 2, a, and b respectively) led to decrease in the zeta potential (less negative). It could be due to the interconnection between Arabic Gum's chains in the particles surface which cause a reduction of negative repulsion force of particles surface while it increased in higher concentration of the NSO (Figure 2, c). Likewise, in the case of Zedo gum, its increase probably due to the antagonistic effect with other wall materials, WPI and AG, influences negatively on zeta potential.

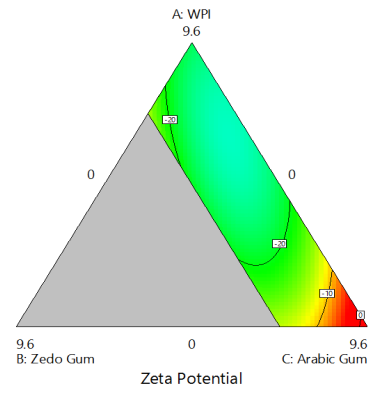

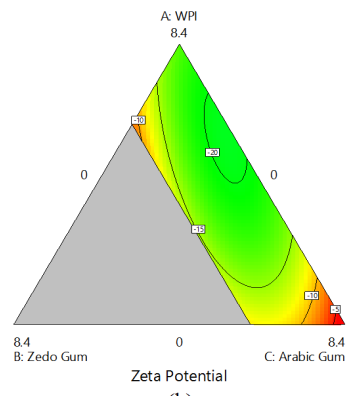

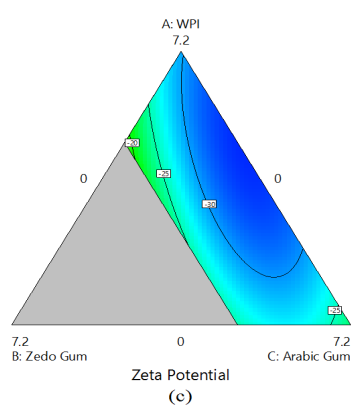

Fig. 2. Contour plots showing the effects of wall materials on the zeta potential of Nigella sativa seed oil microparticles at (a): $20 \%,(b): 30 \%,(c): 40 \%$ oil levels. 


\section{Encapsulation efficiency}

The presence of oil on the powder surface is the most undesirable property of encapsulated powder since this oil is readily susceptible to oxidation. In order to calculate the $\mathrm{EE}(\%), 4 \mathrm{~g}$ of powder placed in a filter paper was washed six times with $10 \mathrm{ml}$-hexane. Then, the solution containing the extracted oil was left to evaporate at room temperature. The surface oil was calculated based on the difference between the initial clean flask and that containing the extracted oil residue. Figure 3 illustrates $\mathrm{EE}(\%)$ as a function of the different wall material concentration. As it can be drawn from all Fig. 3, there is a reverse correlation between oil loading and EE in terms of WPI concentration, which agrees with previous reports. Such decrease in EE is explained by shortening of the diffusion path of oil droplets in the microcapsules. Higher oil loading makes easier diffusion of oil droplet into the particle surface by decreasing the emulsion viscosity. So as a result, the surface oil content of the powder increases which led to a lower encapsulation efficiency. The same behavior was observed
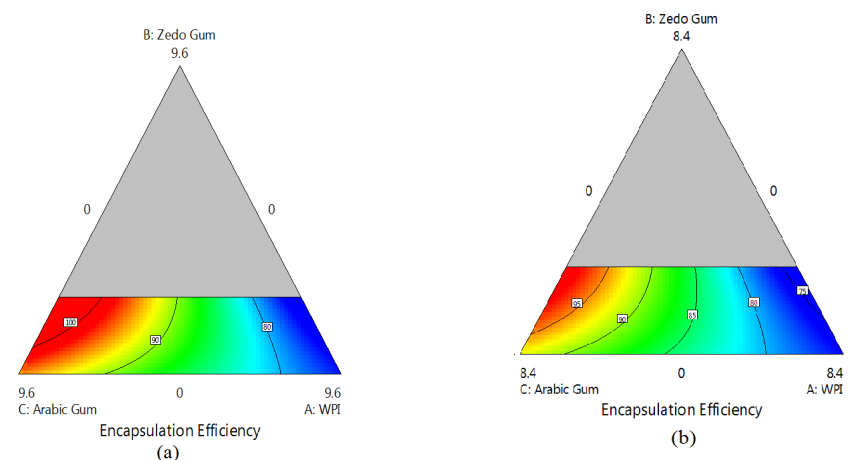

(b)

Fig. 3. Contour plots showing the effects of wall materials on the encapsulation efficiency of encapsulated oil at (a): $20 \%$, (b): $30 \%$, (c): $40 \%$ oil levels.

\section{PDI index}

The PDI value refers to the degree of uniformity of the particle size distribution, and lower amount of it results in smaller distribution [23]. Therefore, low PDI values indicate strong uniformity and consistent processes. Fig 4 shows that the particle dispersion index decreases with increasing both Whey Protein Isolate and Arabic gum. In the case of Arabic gum, the reason for this is that it can adequately cover the particles and thus increase the repulsive force between the particles, which ultimately reduces the particle size distribution. The particle dispersion index has a direct correlation with the increase in Zedo gum concentration, which may be due to the high viscosity of the Zedo gum in higher concentrations. As it is obvious from the figure 4, the particle dispersion index was just significant $(\mathrm{P}<0.05)$ in the 30 percent of the oil. According to Fig. 4, the particle by some researchers[20-22]. It may also be explained by the void volume of individual microcapsules; thereby effective site of the whey protein interacting with oils is too inadequate to interact with high amount of oils which cause leakage of oil to the surface of the microcapsules during or soon after freezing process. Encapsulation efficiency in all concentrations of oil increased with raising the concentration of the Arabic gum. One of the reasons may be due to the high agglomeration of microcapsules with Arabic gum that preserve better core materials and compounds. Plus, Arabic gum keeps the aroma and volatile content better due to its high emulsion properties. Frascareli et al. observed in 2012 that the encapsulation efficiency of the coffee oil increased with enhancing the Arabic gum concentration [21]. As shown in Fig 3, change in the concentration of Zedo gum has not notable effect on the microencapsulation efficiency. There is neither antagonistic nor synergistic interaction between wall materials except Arabic gum that shows partly synergistic property with Zedo gum. dispersion index decreases with increasing the amount of NSO.

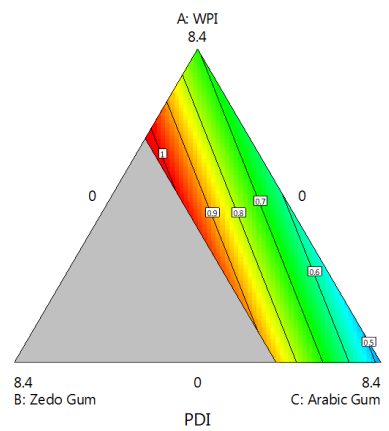

Fig. 4. Contour plot of the effects of wall materials on the particle dispersion index of Nigella sativa seed oil microparticles at $30 \%$ oil level. 


\section{E. Electrical conductivity}

Since the electrical conductivity of water, especially due to the dissolution of electrolytes, is 100 to 1000 times greater than that of oil, this indicator is used to determine the continuous phase of the emulsion. The electrical conductivity of the oil phase is often considered to be zero in comparison with the water phase. Consequently, the reduction of electrical conductivity means the formation of
$\mathrm{O} / \mathrm{W}$ emulsion and the lack of change in electrical conductivity indicates the formation of $\mathrm{W} / \mathrm{O}$ emulsion [24]. The results showed that different concentrations of wall materials have a significant effect on the conductivity of the microcapsules $(\mathrm{P}<0.05)$. Electrical conductivity at all three levels of the oil increased with increasing in the amount of the WPI while both $A G$ and $Z G$ caused a decrease in electrical conductivity.

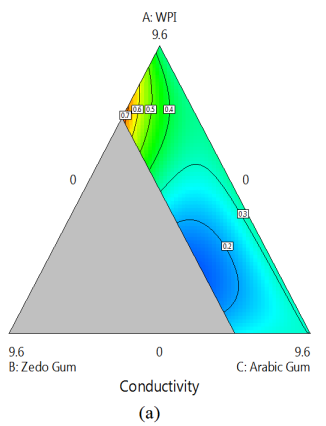

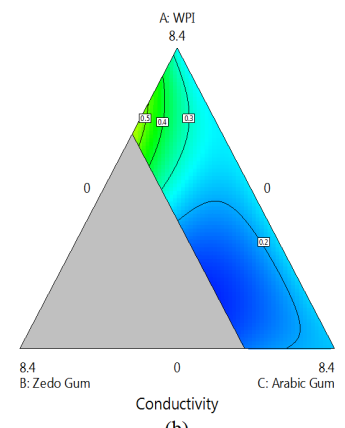

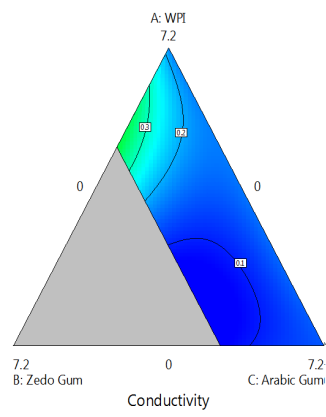

Fig. 5. Contour plots showing the effects of wall materials on the conductivity of encapsulated oil at (a): $20 \%$, (b): $30 \%$, (c): $40 \%$ oil levels.

\section{F. Color parameters}

In order to assess the influence of the investigated variables on the color of N. sativa-loaded microcapsules, the $\mathrm{a}^{*}$ and $\mathrm{b}^{*}$ were reported, as these parameters correspond to the greenness-redness and blueness-yellowness of the fabricated microcapsules containing NSO respectively. In the case of $a^{*}$ index, when the number goes to the negative side, indicating that the color of the food material tends towards the green color, and as far as the positive side is concerned, it indicates that the color of the food substance tends to be red. Increasing the amount of WPI in all three levels of the encapsulated NSO (a: $20 \%$; b: $30 \%$; c: $40 \%$ ) led to decrease in the $a^{*}$ index (Fig 6), which means green color appearance in capsules. The $\mathrm{a}^{*}$ parameter was reported as a function of the WPI/AG/ZG mixture, which got more negative by exceeding amounts of WPI. Increasing the concentration of WPI and AG in all three levels of oil resulted in a decrease in the $b^{*}$ index (Fig 7) while ZG was reversed and increased that. In the case of $b^{*}$, the negative values indicate a blue color and a positive value means yellow. Since the ZG was yellow, but AG was white. As a result, the micro-capsules produced by the $\mathrm{ZG}$ were yellowish.
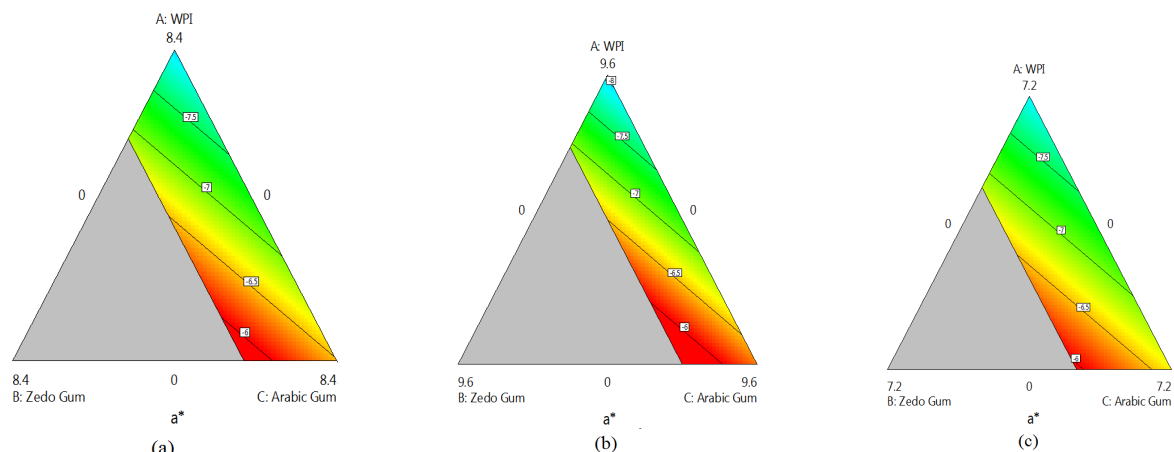

Fig. 6. Contour plots showing the effects of wall materials on the $\mathrm{a}^{*}$ index of microparticles containing Nigella sativa seed oil at (a): $20 \%$, (b): $30 \%$, (c): $40 \%$ oil levels. 


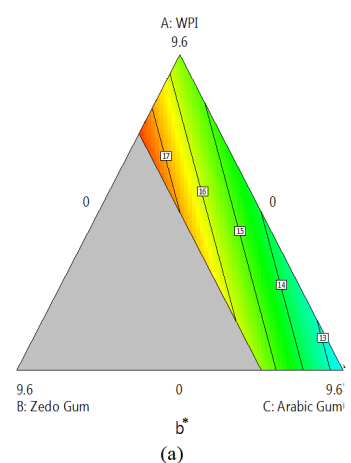

(a)

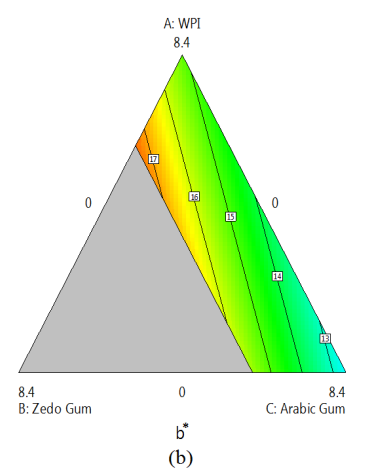

(b)

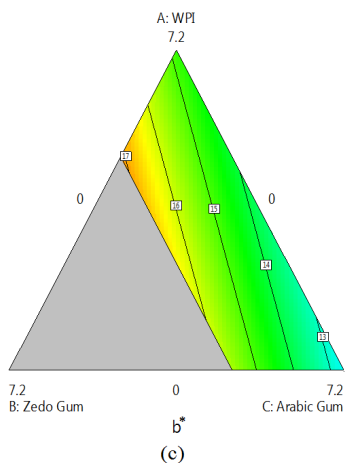

Fig. 7. Contour plots showing the effects of wall materials on the $b^{*}$ index of microparticles containing Nigella sativa seed oil at (a): $20 \%$, (b): $30 \%$, (c): $40 \%$ oil levels.

\section{G. Optimization and desirability}

One of the objectives of this study was to determine the optimal values of the variables to generate microcapsules with the minimum droplet size, PDI, and $\mathrm{a}^{*}$ and with maximal zeta potential, conductivity, encapsulation efficiency, and $\mathrm{b}^{*}$. The desirability function method was employed for the multi-objective optimization. The desirability function for individual responses was estimated through numerical methods, and the total desirability function was calculated. The results of statistical analysis, which led us to finding the optimum variables based on the physical qualities of microcapsules, are presented in Table 3. It is remarkable that the total desirability values of the predicted zone correspond to 0.41. Furthermore, microparticles were produced based on the optimal conditions of wall material components, and eventually the structural and morphological characteristics of the optimized microcapsules were investigated.

TABLE 3.

OPTIMUM ENCAPSULATION CONDITIONS OF NIGELLA SATIVA SEED OIL MICROCAPSULES.

\begin{tabular}{lc}
\hline Factor name & Desirability \\
\hline WPI (\%) & 39.05 \\
\hline ZG (\%) & 4.192 \\
\hline AG (\%) & 16.758 \\
\hline Oil (\%) & 40 \\
\hline Z-average (d.nm) & 876.863 \\
\hline Zeta potential (mV) & -32.441 \\
\hline Encapsulation efficiency (\%) & 80.021 \\
\hline PDI & 0.647 \\
\hline Conductivity & 0.171 \\
\hline $\mathrm{a}^{*}$ & -7.353 \\
\hline $\mathrm{b}^{*}$ & 14.697 \\
\hline Selected desirability (\%) & 0.41 \\
\hline
\end{tabular}

\section{H. FT-IR analysis}

Fig 8 demonstrates the FT-IR spectra of the polymers and the capsule. According to Carbonaro and Nucara (2010), $\alpha$ helix, $\beta$ sheet, random coil and antiparallel sheet are the main structural forms that can be described by the bands amide I and II [25]. It is observed in curve A, Whey Protein Isolate, that a peak at $1656 \mathrm{~cm}^{-1}$ appeared. Generally, the presence of any strong peak in the region of $1630-1680 \mathrm{~cm}^{-1}$ shows strong stretching vibrations of the carboxyl groups $(\mathrm{C}=\mathrm{O})$. The Amide I shows a $\mathrm{C}-\mathrm{N}$ stretching band at $1392 \mathrm{~cm}^{-1}$. Additionally, the Amide I displays a bending band at $608 \mathrm{~cm}^{-1}$ and $1100 \mathrm{~cm}^{-1}$. Therefore, the Amide I exists in the WPI structure. These findings are in agreement with the reports of some researchers [26-28]. Gum Arabic, curve B, represented typical bond at $3418 \mathrm{~cm}^{-1}$ which is representative of $-\mathrm{OH}$ stretching band. In the curve $\mathrm{C}$, Zedo Gum, the characteristic peaks at 2924-2927 $\mathrm{cm}^{-1}$ and $2855 \mathrm{~cm}^{-1}$ correspond to the asymmetric and symmetric stretching vibrations of $-\mathrm{CH} 2$ functional groups, respectively [29].

The FTIR spectra of encapsulated oil shows similar profile with the polymers. Therefore, the FTIR results suggested that the presence of Nigella sativa seed oil into the polymeric matrix was almost stable and did not interact significantly with $\mathrm{WPI} / \mathrm{AG} / \mathrm{ZG}$ bend as matrix components. However, in the fingerprint region, the transmittance bands were detected at $716 \mathrm{~cm}^{-1}$ and $1742 \mathrm{~cm}^{-1}$ revealing the presence of $\mathrm{C}-\mathrm{H}$ bonds and covalent linkage (amid I), respectively. 


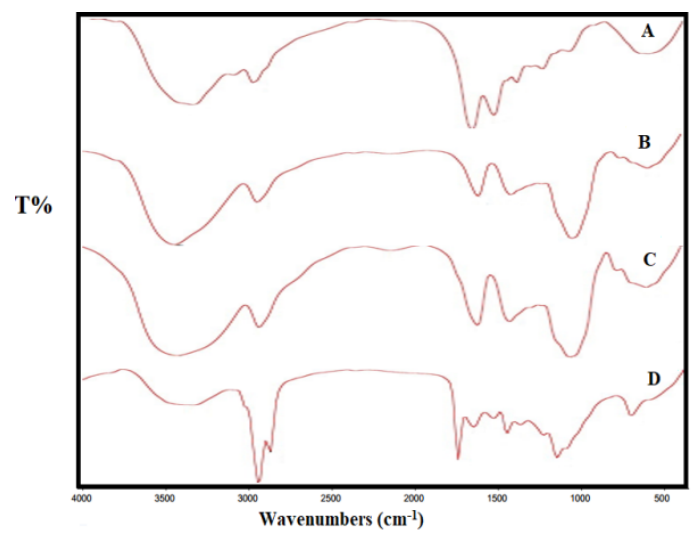

Fig. 8. Fourier-transform infrared spectroscopy (FTIR) spectra of: A, whey protein isolate (WPI); B, arabic gum (AG); C, zedo gum (ZG); D, powdered microcapsules.

\section{Morphology of microcapsules}

The morphology of WPI, AG, ZG, and N. sativa coated microcapsules produced by freeze-drying is presented in Figure 9. The SEM examination was used to study the morphology and size of the capsules provided. The SEM results showed that the microparticles had an irregular structure similar to that of broken glass. The presence of cavities or pores was observed in the walls of the cracks (Figure 9). Forming ice crystals in the emulsion freezing step during the freeze-drying process could have created these cavities [30]. A similar microstructure was observed for wheat germ oil microparticles obtained by freeze-drying and encapsulated with whey protein concentrate (WPC) and maltodextrin [31]. As expected, however, the type of biopolymer used as wall material produced particles with different diameters. The average capsule particle size obtained by the DLS method was almost $876.863 \mathrm{~nm}$, but SEM micrographs showed that the average size was 772.6 $\mathrm{nm}$. These results revealed that the size of the dried emulsion particles was smaller than that of the optimization samples.

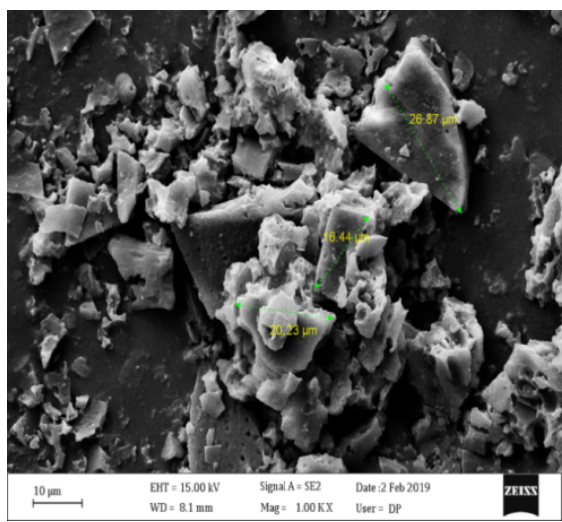

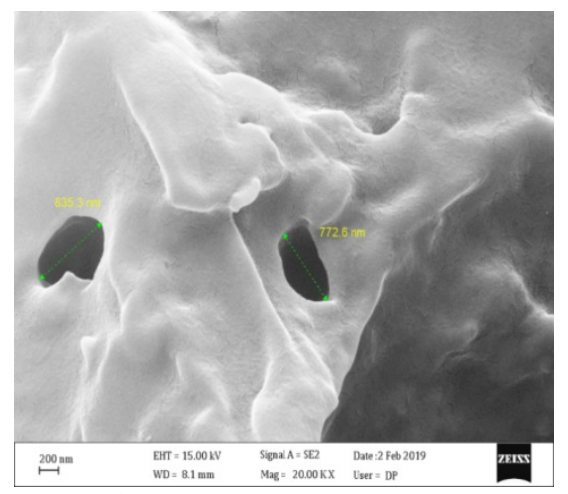

Fig. 9. Scanning electron microscopy (SEM) micrographs of freeze-dried powders of Nigella sativa seed oil microcapsules stabilized by whey protein isolate (WPI), arabic gum (AG), and zedo gum $(\mathrm{ZG})$.

\section{CONCLUSION}

Freeze-drying encapsulation of Nigella sativa seed oil, using WPI, $A G$, and $\mathrm{ZG}$ as wall materials, led to the formation of fine kinetically stable emulsion. The findings demonstrated that increase of the Zedo gum concentration had a negative impact on size of the emulsion droplets, with a reduction in the number of droplets. A lower particle size distribution was found with increasing the amount of the WPI and AG. SEM observation was corresponded with the predicted particle size of the optimized microcapsules. The color of microcapsules was directly attributed to the wall materials concentration. FTIR analysis demonstrated that there were no significance interactions in stabilizing NSO by mixing WPI/AG/ZG. The results of these findings suggest encapsulating Nigella sativa seed oil in blending of WPI, $A G$ and $Z G$ as a functional way to generate microparticles containing NSO with reasonably long shelf life that could be of great use in food and pharmaceutical products.

\section{ACKNOWLEDGEMENTS}

This research did not receive any specific grant from funding agencies in the public, commercial, or not-for-profit sectors.

\section{REFERENCES}

[1] Ahmad A, Husain A, Mujeeb M, et al. A review on therapeutic potential of Nigella sativa: A miracle herb. Asian Pacific Journal of Tropical Biomedicine. 2013;3(5):337-352.

[2] Darakhshan S, Bidmeshki Pour A, Hosseinzadeh Colagar A, Sisakhtnezhad S. Thymoquinone and its therapeutic potentials. Pharmacological Research. 2015;95-96:138-158.

[3] Burits M, Bucar F. Antioxidant activity of Nigella sativa essential oil. Phytotherapy Research. 2000;14(5):323-328.

[4] Ceballos AM, Giraldo GI, Orrego CE. Effect of freezing rate on quality parameters of freeze dried soursop fruit pulp. Journal of Food Engineering. 2012;111(2):360-365. 
[5] Kagami Y, Sugimura S, Fujishima N, Matsuda K, Kometani T, Matsumura Y. Oxidative stability, structure, and physical characteristics of microcapsules formed by spray drying of fish oil with protein and dextrin wall materials. Journal of Food Science. 2003;68(7):2248-2255

[6] Krishnan S, Bhosale R, Singhal RS. Microencapsulation of Cardamom oleoresin: Evaluation of blends of gum arabic, maltodextrin and a modified starch as wall materials. Carbohydrate Polymers. 2005;61(1):95-102.

[7] Arzeni C, Martínez K, Zema P, Arias A, Pérez OE, Pilosof AMR. Comparative study of high intensity ultrasound effects on food proteins functionality. Journal of Food Engineering. 2012;108(3):463-472.

[8] Klein M, Aserin A, Svitov I, Garti N. Enhanced stabilization of cloudy emulsions with gum arabic and whey protein isolate. Colloids and Surfaces B: Biointerfaces. 2010;77(1):75-81.

[9] Fadavi G, Mohammadifar M, Zargaran A, Azadnia E. The study of composition, molecular weight and rheological characteristics of zedo gum exudates from Amygdalus scoparia. Iranian Journal of Nutrition Sciences \& Food Technology. 2013;7(5).

[10] Karimi Sani I, Alizadeh M, Pirsa S, Moghaddas Kia E. Impact of operating parameters and wall material components on the characteristics of microencapsulated Melissa officinalis essential oil. Flavour and Fragrance Journal. 2019;34(2):104-112.

[11] Mohammadi A, Jafari SM, Esfanjani AF, Akhavan S. Application of nano-encapsulated olive leaf extract in controlling the oxidative stability of soybean oil. Food Chemistry. 2016;190:513-519.

[12] Khoshmanzar m, Ghanbarzadeh b, Hamishehkar h, Sowti m, Rezayi mr. Investigation of effective parameters on particle size, zeta potential and steady rheological properties of colloidal system based on carrageenan-caseinate nanoparticles. 2012.

[13] Esfanjani AF, Jafari SM, Assadpoor E, Mohammadi A. Nanoencapsulation of saffron extract through double-layered multiple emulsions of pectin and whey protein concentrate. Journal of Food Engineering. 2015;165:149-155.

[14] Silva VM, Vieira GS, Hubinger MD. Influence of different combinations of wall materials and homogenisation pressure on the microencapsulation of green coffee oil by spray drying. Food Research International. 2014;61:132-143.

[15] Silva EK, Azevedo VM, Cunha RL, Hubinger MD, Meireles MAA. Ultrasound-assisted encapsulation of annatto seed oil: Whey protein isolate versus modified starch. Food Hydrocolloids. 2016;56:71-83.

[16] Randall RC, Phillips GO, Williams PA. The role of the proteinaceous component on the emulsifying properties of gum arabic. Food Hydrocolloids. 1988;2(2):131-140.

[17] Joye IJ, McClements DJ. Production of nanoparticles by antisolvent precipitation for use in food systems. Trends in Food Science \& Technology. 2013;34(2):109-123.
[18] Kakran M, Sahoo NG, Tan IL, Li L. Preparation of nanoparticles of poorly water-soluble antioxidant curcumin by antisolvent precipitation methods. Journal of Nanoparticle Research. 2012;14(3):757.

[19] Haghju S, Beigzadeh S, Almasi H, Hamishehkar H. Chitosan films incorporated with nettle (Urtica dioica L.) extract-loaded nanoliposomes: I. Physicochemical characterisation and antimicrobial properties. Journal of Microencapsulation. 2016;33(5):438-448

[20] Ahn J-H, Kim Y-P, Lee Y-M, Seo E-M, Lee K-W, Kim H-S. Optimization of microencapsulation of seed oil by response surface methodology. Food Chemistry. 2008;107(1):98-105.

[21] Frascareli EC, Silva VM, Tonon RV, Hubinger MD. Effect of process conditions on the microencapsulation of coffee oil by spray drying. Food and Bioproducts Processing. 2012;90(3):413424.

[22] Tan LH, Chan LW, Heng PWS. Effect of oil loading on microspheres produced by spray drying. Journal of Microencapsulation. 2005;22(3):253-259.

[23] Dubey RR, Parikh RH. Two-stage optimization process for formulation of chitosan microspheres. AAPS PharmSciTech. 2009;5(1):20.

[24] Nielloud F. Pharmaceutical emulsions and suspensions: Revised and expanded. 2000

[25] Carbonaro M, Nucara A. Secondary structure of food proteins by Fourier transform spectroscopy in the mid-infrared region. Amino Acids. 2010;38(3):679-690.

[26] Beaulieu L, Savoie L, Paquin P, Subirade M. Elaboration and Characterization of Whey Protein Beads by an Emulsification/Cold Gelation Process: Application for the Protection of Retinol. Biomacromolecules. 2002;3(2):239-248.

[27] Byler DM, Susi H. Examination of the secondary structure of proteins by deconvolved FTIR spectra. Biopolymers. 1986;25(3):469-487.

[28] Clark AH, Saunderson DHP, Suggett A. INFRARED AND LASER-RAMAN SPECTROSCOPIC STUDIES OF THERMALLY-INDUCED GLOBULAR PROTEIN GELS International Journal of Peptide and Protein Research. 1981;17(3):353-364.

[29] Fadavi G, Mohammadifar MA, Zargarran A, Mortazavian AM, Komeili R. Composition and physicochemical properties of Zedo gum exudates from Amygdalus scoparia. Carbohydrate Polymers. 2014;101:1074-1080.

[30] Anandharamakrishnan C, Rielly CD, Stapley AGF. Spray-freezedrying of whey proteins at sub-atmospheric pressures. Dairy Science \& Technology. 2010;90(2):321-334.

[31] Yazicioglu B, Sahin S, Sumnu G. Microencapsulation of wheat germ oil. Journal of Food Science and Technology. 2015;52(6):3590-3597. 